\title{
Birth of Compound Numbers
}

\author{
Ranjit Biswas* \\ Department of Computer Science, Jamia Hamdard University, Hamdard Nagar, New Delhi, INDIA \\ *Corresponding author: ranjitbiswas@yahoo.com
}

Received October 15, 2014; Revised November 18, 2014; Accepted November 26, 2014

\begin{abstract}
In this paper the author introduces a new kind of numbers called by 'Compound Numbers'. A region R may or may not have imaginary object. A region even may have more than one imaginary objects too. Corresponding to an imaginary object (if exists) of a region R, we get compound objects for the region R. Imaginary objects and compound objects of a region $\mathrm{R}$ are not members of $\mathrm{R}$ and so they are called imaginary with respect to the region R only (i.e. it is a local characteristics property with respect to the region concerned), as they could be core members of another region. Every region has its own set of imaginary numbers (if exist). As a particular instance, the compound objects of the set of real numbers are the complex numbers (of existing concept). In this paper the author discovers imaginary objects of the region $\mathrm{C}$ (the set of complex numbers). The compound objects of $\mathrm{C}$ are called by 'compound numbers'. Collection of all compound numbers is denoted by the set $\mathrm{E}$. This work just reports the birth of compound numbers, not further details at this stage. It is claimed that "Theory of Numbers" will get a new direction by the birth of compound numbers. A new "Theory of Objects' and the classical "Theory of Numbers" as a special case of it were also studied in [16]. In this paper we say that every complete region has its own 'Theory of Numbers', where the classical 'theory of numbers' is just a special instance corresponding to a particular complete region RR. Consequently, we also introduce a new field called by "Object Geometry" of a complete region, being a generalization of our classical geometry of the existing style, from elementary to the higher level.
\end{abstract}

Keywords: region, im-object, im-number, rim, cim, compound object, compound number, onteger

Cite This Article: Ranjit Biswas, "Birth of Compound Numbers." Turkish Journal of Analysis and Number Theory, vol. 2, no. 6 (2014): 208-219. doi: 10.12691/tjant-2-6-4.

\section{Introduction}

It is observed and explained with several examples in [16], with rigorous analysis and justifications, that most of the simple and useful results, equalities, identities, formulas, laws, rules etc. which are frequently practiced at secondary school level of mathematics or higher level are not valid (i.e. can not be verified) in groups, rings, modules, fields, linear spaces, algebra over a field, associative algebra over a field, division algebra or in any existing standard algebraic system, in general, by virtue of their own characteristic properties only. This is a major gap and/or incompleteness in the existing literatures while regarding 'Algebra' as a subject. This invokes us to discover the exact minimum algebra which has the potential to provide a complete and sound platform in this sense. Quite naturally one has to think of a permutation / combination of the various existing algebraic structures to unearth a possible identity of a platform 'algebraic system' in which the useful standard results of elementary algebra or higher algebra can be verified (can be accepted to be valid). Attention needs to be given to explore and rigorously study this hidden algebraic system considering its unique potential to provide a complete and sound platform, a minimal platform, on which the simple and useful results, equalities, identities, formulas, laws, rules etc. of elementary/higher algebra, which are frequently practiced at secondary school level of mathematics or higher level etc. can be verified (recognized to be valid). In quest of identifying this important unknown platform, a new algebraic system called by "Region" is developed in independently with a unique identity, and its important properties are explored to view and understand how does it validate the simple and useful results, equalities, identities, formulas, laws, rules etc. of elementary/higher algebra, whereas they are not valid (i.e. can not be verified) in groups, rings, modules, fields, linear spaces, algebra over a field, associative algebra over a field, division algebra or in any existing standard algebraic system, in general. The properties of the regions are interesting and this is the only minimal algebra which justifies free and fluent practice of elementary as well as higher algebra. This important identification was missing so far in any past literature of algebra or mathematics, and surely the region algebra is a unique algebra of absolute integrated nature. A new algebraic theory called by "Theory of Objects" is introduced which generates the notion of 'imaginary objects' in region algebra. The classical imaginary numbers (or, complex numbers) are just one particular instance of the imaginary objects. It is discovered that like the imaginary number i corresponding to the set $\mathrm{R}$ of real numbers, there exist imaginary number e corresponding to the set $\mathrm{C}$ of complex numbers. Consequently, a new theory of numbers is discovered 
called by 'Theory of Compound Numbers'. It is sure that the algebraic "Theory of Objects" will play a huge role to the Number Theorists in a new direction.

\section{Region Algebra: The Minimal Platform Algebra}

Algebra is regarded as one of the most beautiful branches of mathematics and it is about finding the unknowns. It tastes to be both dry and juicy. It is shown in this chapter by a number of examples that most of the simple and useful results, identities/equalities, formulas or algebraic expressions or equations of elementary algebra (commonly practiced at secondary school level of mathematics) are not valid (can not be computed/verified) in general in the existing standard algebraic systems like: group, ring, field, module, linear space, algebra over a field, associative algebra over a field, division algebra, etc. (by the phrase: "the result is valid in the algebraic system A", we mean here that the result can be successfully computed and established in the algebraic system A). Consequently, it is unearthed that there was a major gap lying hidden so far in the subject "Algebra". To bridge this gap the author introduces a new algebraic system called by "Region", independently in a unique way. The huge potential and strength of this unique algebraic system is lying in the fact that it is the minimal algebra which validates (i.e. can be verified in it) the results, equalities, identities, formulas, laws, rules etc. of elementary/higher algebra, which are frequently practiced at secondary school level of mathematics or higher level are not valid. Region provides the minimal platform on which all elementary algebraic computations practiced by students, teachers, mathematicians, scientists, engineers, etc. are done. Such a complete and sound platform for 'elementary algebra' can not be provided by any existing algebraic system like: group, ring, module, field, linear space, algebra over a field, associative algebra over a field, division algebra, etc. in general. This important fact was hidden so far to the algebraists, and has been unearthed here. With this philosophy, it can be realized that all the existing classical algebraic systems are weaker than the algebraic system 'region'. An initial development of 'The theory of regions' is done with a lot of characterizations, establishing a number of important properties of the regions. We present the theory of "Regions" [16] in brief here which will be required to explore the birth of compound numbers.

\section{Note 2.1.}

Throughout our discussion in this work, the following standard definitions are followed by us for (i) 'K-Algebra', (ii) 'Associative Algebra over a field $\mathrm{K}$ ', and for (iii) 'Division Algebra':

(i) An 'Algebra over a field K' (or, a K-Algebra) is a vector space $A$ over $\mathrm{K}$ equipped with a compatible notion of multiplication

$$
\in \text { A. }
$$$$
(\mathrm{a} \bullet \mathrm{x}) *(\mathrm{~b} \bullet \mathrm{y})=(\mathrm{a} . \mathrm{b}) \bullet(\mathrm{x} * \mathrm{y}), \forall \mathrm{a}, \mathrm{b} \in \mathrm{K} \text { and } \forall \mathrm{x}, \mathrm{y}
$$

(ii) An 'Associative Algebra over a field $\mathrm{K}$ ' is a vector space over $\mathrm{K}$ which also allows the multiplication of vectors in a distributive and associative manner, having bilinearity of the multiplication. (iii) A 'Division Algebra' $\left(\mathrm{D},+,{ }^{*}\right)$ is a set $\mathrm{D}$ together with two binary operations such that it is a unit ring and also (D- $\{0\},{ }^{*}$ ) forms a group. Thus a division algebra D allows division operation by non-zero elements, but $\mathrm{D}$ need not be commutative with respect to its multiplication operation. A division algebra must contain at least two elements. In general a division algebra is not a field. A commutative division algebra is a Field. A division algebra is not necessarily a K-algebra and also is not necessarily an Associative Algebra.

\subsection{Justification behind the Genuine need to Define/identify a new Algebraic System}

A system consisting of a non-null set $\mathrm{S}$ and one or more n-ary operations on the set $\mathrm{S}$ is called an algebraic system, denoted by the notation $\left(\mathrm{S}, \mathrm{O}_{1}, \mathrm{O}_{2}, \ldots, \mathrm{O}_{\mathrm{r}}\right)$ where $\mathrm{O}_{i}, \mathrm{i}=1,2, \ldots, \mathrm{r}$, are operations on $\mathrm{S}$. An algebraist can define an infinite number of new algebraic systems. The objective of this present work is NOT just to define a new algebraic system, but to recognize and identify a major gap of the subject 'Algebra' lying hidden so far; and then to open a new direction to visualize the 'Theory of Numbers' in a different way. In this section we show that the existing algebraic systems viz. groups, rings, modules, fields, linear spaces, algebra over a field, associative algebra over a field, division algebra or any existing standard algebraic system, in general, are not sufficient to provide a sound and complete environment/platform or algebraic right/validity to the mathematicians for performing many simple algebraic computations, for establishing many useful and simple identities or equalities of two algebraic expressions, and for establishing many useful algebraic results/solutions etc. of elementary algebra; although many of these results/equalities/identities are well known to and being practiced by the secondary school students.

We begin here with a collection of few cases or issues (out of infinite number) on the various standard algebraic systems: groups, rings, modules, fields, etc. These cases (five cases) are mentioned below for the sake of instance only, although they are no doubt very simple and obvious cases to any algebraist. But special attention of the readers is required on the situations presented in Case-2,3,4,5. Then we justify the genuine needs for identifying a new kind of atomic, well complete, sound and unique algebraic system in an independent way with its self-identity.

\section{Case 1.}

If an expression like $\mathrm{x}+2 . \mathrm{y} \oplus \mathrm{z}$ is a valid expression in an algebraic system $\mathrm{A}$ where $\mathrm{x}, \mathrm{y}, \mathrm{z} \in \mathrm{A}$, then one can immediately say that A can not be just a group or a ring or a field or a linear space in general. However, it could be an 'Associative Algebra over a field', or something else.

Consider the equality (identity): $(x+y)^{2}=x^{2}+2 . x * y$ $+\mathrm{y}^{2}$ which is an absurd equality (as it can not be verified) in general in a group or in a ring/module or in a field or in a linear space or in an associative algebra over a field, but can be well verified in some 'algebra over some field'. Here it may be noted that the LHS of this equality can be evaluated in a ring or in a field, but not the RHS (assuming that the notation $\mathrm{t}^{2}$ stands for the expression $\mathrm{t} * \mathrm{t}$ ).

Now consider few quite interesting cases below (Case-2,3,4,5): 


\section{Case 2.}

A very simple example from elementary algebra, very frequently used by the secondary school students, is the equality (identity) of type given by $\left(\frac{2}{3}\right)\left(\frac{x}{y}\right)=\frac{2 x}{3 y}$.

But this identity can 'not be verified' in general in a group, ring, module, field, linear space, 'algebra over a field (i.e. K-algebra)', 'associative algebra over a field', Division Algebra, or in any standard algebraic system.

It is because of the reason that:

(i) since division is involved, it can not be a simple ' $\mathrm{K}$ algebra', in general.

(ii) if it is just a simple division algebra, then see that $\left(\frac{2}{3}\right)\left(\frac{x}{y}\right)=\left(2 \cdot \frac{1}{3}\right) \bullet\left(x^{*} \mathrm{y}^{-1}\right)$ is correct, but the deduction $(2$. $\left.\frac{1}{3}\right) \bullet\left(x^{*} y^{-1}\right)=(2 \bullet x) *\left(\frac{1}{3} \bullet y^{-1}\right)$ is not guaranteed. Hence, $\left(\frac{2}{3}\right)\left(\frac{x}{y}\right) \neq \frac{2 x}{3 y}$ in general in a division algebra.

\section{Case 3.}

Because of the similar reason as in Example 5.1 above, it can be observed that if an equality (in fact it is an identity) of type given by

$$
\begin{aligned}
& (a \bullet x \oplus 1 /(b \bullet y))^{2}= \\
& a^{2} \cdot x^{2} \oplus 1 /\left(b^{2} \cdot y^{2}\right) \oplus(2 a / b) \bullet(x / y),
\end{aligned}
$$

is known to be a valid identity (i.e. can be computed and verified) in an algebraic system $A$ where $x, y \in A$, $a$ and $b$ are members (scalars) of some field F, then it can be observed that none of the following statements are true in general:

(i) A is just an 'algebra over a field K' (K-algebra), not more.

(ii) A is just an 'associative algebra over a field', not more.

(iii) A is just a Division Algebra, nothing more.

\section{Case 4.}

By a careful observation it can be seen that even a simple computation of 'cross-multiplication' of secondary school level elementary algebra like: if $\frac{2 x}{7 y}=\frac{5 z}{3 t}$ then $6 x t$ $=35 \mathrm{yz}$ (and conversely), can 'not be verified' in general in an 'algebra over a field (i.e. K-algebra)', 'associative algebra over a field', Division Algebra, or in any standard algebraic system (assuming that division by zero element is not allowed).

It is because of the reason that:

(i) Since division is involved, it can not be a simple ' $\mathrm{K}$ algebra' only.

(ii) Suppose that it is just a simple division algebra by definition as mentioned in Note 2.1 (without carrying any additional properties). Then we see the following fact: the equality $\frac{2 x}{7 y}=\frac{5 z}{3 t}$ implies that $(2 \mathrm{x})(7 \mathrm{y})^{-1}=(5 \mathrm{z})(3 \mathrm{t})^{-1}$; and then, the equality $(2 x)(7 y)^{-1}=(5 z)(3 t)^{-1}$ implies, after few computing steps, that $(2 x)(3 t)=(5 z)(7 y)$.
But the equality $(2 \mathrm{x})(3 \mathrm{t})=(5 \mathrm{z})(7 \mathrm{y})$ does not imply that $6 \mathrm{xt}=35 \mathrm{yz}$, by definition as in Note 2.1 .

Thus there is no guarantee that a given division algebra will satisfy the 'cross-multiplication' property by virtue of the definition of division algebra.

\section{Case 5.}

By definition of division algebra, there is no guarantee that a very simple square identity like:

$$
(3 \mathrm{x} / 7 \mathrm{y})^{2}=\left(9 \mathrm{x}^{2} / 49 \mathrm{y}^{2}\right)
$$

can be validated in a division algebra.

Then, the immediate question that arises to us is: "What could be the minimal algebraic system in which the above identities or cross multiplication results, etc are valid (i.e. can be verified)?".

For a possible answer, an algebraist has to think of a permutation/combination of the various existing algebraic structures to make out a possible identity of A. But, he might seek to make a unique identity for this algebraic system A defined in an independent and atomic way, and then to study the various properties of $\mathrm{A}$, various results valid on $\mathrm{A}$, highlighting its unique importance/role in Algebra. It is because of the reason that this algebraic system A is supposed to be the most appropriate and needful minimal platform for practicing the problems of elementary algebra of secondary school level, compared to any other existing standard algebraic system, in general.

Consequently we feel that there a genuine need to identify that algebraic system, which is hidden so far, unrecognized so far, but a very powerful algebraic system in the sense that it can provide the actual and minimal base-platform for the subject 'elementary algebra'.

\section{Note}

We at this moment are studying the above cases strictly by the definition of Division Algebra (NOT recollecting the existing examples of division algebra, because the examples of division algebra at this point may create confusion to the breaking philosophy behind the genuine requirement to identify the minimal platform algebra for the mathematicians). It is because of the unearthed fact that many of the existing examples of division algebra where the above cases (Case-2,3,4,5) can be verified are not just as per definition of division algebra, but a lot more. These examples carry with them few in-built additional characteristic properties which are not provided by the definition of division algebra. The region algebra is the minimum requirement for validity of the above cases, and many such cases. One could visualize the location of "Region" as mentioned below:-

Group $\rightarrow$ Ring $\rightarrow$ Field $\rightarrow$ Linear Space $\rightarrow$ Division Algebra $\rightarrow$ Region Algebra.

\subsection{Introducing a New Algebraic System 'Region'}

It has been observed that there is a genuine need to introduce a new algebraic system having unique selfidentity in order to provide a minimal but sufficient platform where 'elementary mathematics' can be fluently practiced with algebraic right and validity. This job is done in [16] by introducing the algebraic system called by "Region", which is a very simple algebraic system, very 
complete and sound. We produce below the same with a further rigorous study and analysis, and then present important applications of regions in the subsequent chapters.

\section{Definition 2.1 Region}

Consider a non-null set A equipped with three binary operations $\oplus, *$ and $\bullet$ such that for a given field $(\mathrm{F},+,$.$) ,$ the following three conditions are satisfied: -

(i) $(\mathrm{A}, \oplus, *)$ forms a field,

(ii) $(\mathrm{A}, \oplus, \bullet)$ forms a linear space over the field $(\mathrm{F}$, $+,$.$) , and$

(iii) A satisfies the property of: "Compatibility with the scalars of the field F"

i.e. $(\mathrm{a} \bullet \mathrm{x}) *(\mathrm{~b} \bullet \mathrm{y})=(\mathrm{a} . \mathrm{b}) \bullet(\mathrm{x} * \mathrm{y}) \forall \mathrm{a}, \mathrm{b} \in \mathrm{F}$ and $\forall \mathrm{x}, \mathrm{y}$ $\in$ A.

Then the algebraic system $\left(\mathrm{A}, \oplus,,^{*}, \bullet\right)$ is called a Region over the field $(\mathrm{F},+,$.$) .$

If there is no confusion, we may simply use the notation A to represent the region $\left(\mathrm{A}, \oplus,{ }^{*}, \bullet\right)$, for brevity.

\section{Definition 2.1.1. Inner Field, Outer Field, Base Field}

The field $\left(\mathrm{A}, \oplus,{ }^{*}\right)$ is called the "inner field" of the region $(\mathrm{A}, \oplus, *, \bullet)$; and the field $(\mathrm{F},+,$.$) of the linear space$ $(\mathrm{A}, \oplus, \bullet)$ is called the "outer field" or the "base field" of the region $(\mathrm{A}, \oplus, *, \bullet)$.

\section{Definition 2.1.2. First Multiplication Operation, Second Multiplication Operation, Third Multiplication Operation}

The sequence of the three operations “ $\oplus$ ”, “*”, and “•” appearing in the notation $(\mathrm{A}, \oplus, *, \bullet)$ representing the region $\mathrm{A}$ is important in the sense that the operation "*" of the region $A$ which is the multiplication operation of the inner field $(\mathrm{A}, \oplus, *)$ is called the "first multiplication" operation of the region $\mathrm{A}$; whereas the operation "•" of the region $\mathrm{A}$ which is the multiplication operation of the linear space $(\mathrm{A}, \oplus, \bullet)$ is called the "second multiplication" operation of the region A. The multiplication operation "." of the base field F is called the "third multiplication" operation or the "base multiplication" operation of the region A.

\section{Definition 2.1.3. First Addition Operation, Second Addition Operation, Third Addition Operation}

The operation " $\oplus$ " of the region $(\mathrm{A}, \oplus, *, \bullet)$ which is the addition operation of the inner field $(\mathrm{A}, \oplus, *)$ as well as of the linear space $(\mathrm{A}, \oplus, \bullet)$ is called the "first addition" operation of the region $\mathrm{A}$; whereas the operation "+" which is the addition operation of the base field $(\mathrm{F},+,$. is called the "third addition" operation or the "base addition" operation of the region A. (There is no terminology like "second addition" operation of a region A).

Thus in a region A, we deal with two addition operations and three multiplication operations, in general. It is obvious from the definition that a region A must have at least two elements. It may also be noted that every region is an 'algebraic system over a field', but the converse is not true in general.

In the region $(\mathrm{A}, \oplus, *, \bullet)$, its component algebraic system $(\mathrm{A}, \oplus, *)$ is a field. Thus we see that the region $\mathrm{A}$ is a commutative division algebra. Also the other component algebraic system $(\mathrm{A}, \oplus, \bullet)$ is a linear space over the field $\mathrm{F}$. Considering the distributive properties of the field $(\mathrm{A}, \oplus, *)$ along with the condition(iii) of the definition 2.1, it is observed that the region A is F-algebra. Thus a region is a "Commutative Division F-Algebra", but defined independently and uniquely with a self-identity here, with its important properties and results.

\section{What is the importance of 'Region Algebra"?}

We give a huge attention and importance to the "region algebra' because of the so far hidden fact that it is the minimal algebra which permits the mathematicians to practice mathematics in natural fluency (from elementary mathematics to higher level). It is not just a Division Algebra in general (as explained with examples in Case2,3,4,5 earlier in subsection-2.1).

\section{Example 2.1.}

Let $\mathrm{R}$ be the set of real numbers, ' + ' be the ordinary addition operation in $\mathrm{R}$ and ' ' be the ordinary multiplication operation in $\mathrm{R}$. Consider the field $(\mathrm{R},+,$.$) of$ real numbers, and the linear space $(\mathrm{R},+$, .) over the field $(\mathrm{R},+,$.$) . Then the algebraic system (\mathrm{R},+, . .$.$) forms a$ region over the outer field $(\mathrm{R},+,$.$) . This region (\mathrm{R},+, . .$. plays a very important role in our daily life computations, in particular in school level elementary algebra/arithmetic. The content of the syllabus and corresponding instructions at school level algebra is based on the platform of this region $(\mathrm{R},+, . .$.$) , not on the platform of any standard$ algebraic structure like groups, rings, fields, linear spaces, algebra over a field, associative algebra over a field, division algebra or any existing algebraic system, in general. Let us name this region $(\mathrm{R},+, .,$.$) in short by$ "RR".

\section{Definition 2.1.4. "Additive Identity" element of a Region}

The additive identity element of the inner field $(\mathrm{A}, \oplus$, *) of a region $(\mathrm{A}, \oplus, *, \bullet)$ is called the 'additive identity' element of the region $\mathrm{A}$, and is denoted by the notation $0_{\mathrm{A}}$.

Obviously, the 'additive identity' element of a region A is unique [by virtue of inheritance from the properties of the field $(\mathrm{A}, \oplus, *)]$. The additive identity of a region $\mathrm{A}$ is also called the 'zero element' of the region A.

It is obvious that the zero-element of the linear space (A, $\oplus, \bullet)$ and $0_{\mathrm{A}}$, the zero element of the region $\mathrm{A}$, both are the same element.

\section{Definition 2.1.5. "Multiplicative Identity" element of a Region}

The multiplicative identity element of the inner field (A, $\oplus, *)$ of a region $(\mathrm{A}, \oplus, *,-)$ ) is called the 'multiplicative identity' element of the region $\mathrm{A}$ and is denoted by the notation $1_{\mathrm{A}}$.

Obviously, 'multiplicative identity' element of a region $\mathrm{A}$ is unique [by virtue of inheritance from the properties of the field $(\mathrm{A}, \oplus, *)]$. The multiplicative identity of a region $\mathrm{A}$ is also called the 'unit element' of the region $\mathrm{A}$.

Definition 2.1.6. "Additive Inverse" of an element of a Region 
For an element $\mathrm{x}$ of a region $(\mathrm{A}, \oplus, *, \bullet)$ over the field $(\mathrm{F},+,$.$) , the 'additive inverse' of \mathrm{x}$ is defined to be that element of the region $\mathrm{A}$ which is the additive inverse of $\mathrm{x}$ in the inner field $(\mathrm{A}, \oplus, *)$, and is denoted by the notation $\sim \mathrm{x}$.

Obviously, 'additive inverse' of an element of a region is unique [by virtue of inheritance from the properties of the field $(\mathrm{A}, \oplus, *)]$.

\section{Definition 2.1.7. "Multiplicative Inverse" of an element of a Region}

For a non-zero element $\mathrm{x}$ of a region $(\mathrm{A}, \oplus, *, \bullet)$ over the field $(\mathrm{F},+$,$) , the 'multiplicative inverse' of \mathrm{x}$ is defined to be that element of the region $\mathrm{A}$ which is the multiplicative inverse of $\mathrm{x}$ in the inner field $(\mathrm{A}, \oplus, *)$, and is denoted by the notation $\mathrm{x}^{-1}$.

Obviously, 'multiplicative inverse' of an element of a region is unique [by virtue of inheritance from the properties of the field $(\mathrm{A}, \oplus, *)]$. It may be observed that "multiplicative inverse" $\mathrm{x}^{-1}$ of an element $\mathrm{x}$ of a region $\mathrm{A}$ is w.r.t the first multiplication operation of the region $\mathrm{A}$. There is no multiplicative inverse of an element $\mathrm{x}$ of the region $\mathrm{A}$ w.r.t. the second multiplication operation ' $\bullet$ ' and w.r.t the third multiplication operation ' $’$ '

\section{Definition 2.1.8. Four types of Division in a Region}

Let $(\mathrm{A}, \oplus, *, \bullet)$ be a region over the field $(\mathrm{F},+,$.$) .$ There are four types of division can be performed in this algebraic system which are mentioned below.

For all these four types of division, we use a common notation/style like $\frac{\text { numerator }}{\text { deno min ator }}$, (assuming that there is no confusion).

Type (i) Division of an element of the region $A$ by another element of the region $A$

$\forall \mathrm{x}, \mathrm{y}\left(\neq 0_{\mathrm{A}}\right) \in$ region $\mathrm{A}$, the division of the element $\mathrm{x}$ by the non-zero element $\mathrm{y}$ is denoted by the notation $\frac{x}{y}$, and is defined by $\frac{x}{y}=\mathrm{x} * \mathrm{y}^{-1}$.

Replacing $\mathrm{x}$ by $1_{\mathrm{A}}$ and $\mathrm{y}$ by $\mathrm{x}$ in the above, we get the result $\frac{1_{A}}{x}=\mathrm{x}^{-1}\left(\right.$ where $\left.\mathrm{x} \neq 0_{A}\right)$.

Type (ii) Division of an element of the region $A$ by an element of the outer field $F$

$\forall \mathrm{x} \in \mathrm{A}$ and $\forall \mathrm{a}\left(\neq 0_{\mathrm{F}}\right) \in \mathrm{F}$, the division of the region element $\mathrm{x}$ by the field element $\mathrm{a}$ is denoted by $\frac{x}{a}$, and is defined by $\frac{x}{a}=\mathrm{a}^{-1} \bullet \mathrm{x}$.

Replacing a by $1_{\mathrm{F}}$, we get the result $\frac{x}{1_{F}}=\mathrm{x}$.

Type (iii) Division of an element of the outer field $F$ by an element of the region $A$ $\forall \mathrm{a} \in \mathrm{F}$ and $\forall \mathrm{x}\left(\neq 0_{\mathrm{A}}\right) \in \mathrm{A}$, the division of the field element a by the region element $\mathrm{x}$ is denoted by $\frac{a}{x}$, and is defined by $\frac{a}{x}=\mathrm{a} \cdot \mathrm{x}^{-1}$.

Replacing a by $1_{\mathrm{F}}$, we get the result $\frac{1_{F}}{x}=\mathrm{x}^{-1}$.

Type (iv) Division of an element of the outer field $F$ by another element of the outer field $F$.

In the field $(\mathrm{F},+,$.$) , it is known (in field theory) that \forall \mathrm{a}$, $\mathrm{b}\left(\neq 0_{\mathrm{F}}\right) \in \mathrm{F}$, the division of the element a by the non-zero element $\mathrm{b}$ is denoted by the notation $\frac{a}{b}$, and is defined by $\frac{a}{b}=\mathrm{a} \cdot \mathrm{b}^{-1}$

\subsection{Associativity Properties of Regions}

The following three associative properties have been proved in [16] to hold good in a region $(\mathrm{A}, \oplus, *, \bullet)$ over the field $(\mathrm{F},+,$.$) . They are called "No-Scalar Associative$ Property", "One-Scalar Associative Property" and "TwoScalars Associative Property" respectively.

(i) $\mathrm{x} *(\mathrm{y} * \mathrm{z})=(\mathrm{x} * \mathrm{y}) * \mathrm{z}$ : (No-Scalar Associative Property)

(ii) $\mathrm{a} \bullet(\mathrm{x} * \mathrm{y})=(\mathrm{a} \bullet \mathrm{x}) * \mathrm{y}:($ One-Scalar Associative Property)

(iii) $(\mathrm{a} . \mathrm{b}) \bullet \mathrm{x}=\mathrm{a} \bullet(\mathrm{b} \bullet \mathrm{x})$ : (Two-Scalars Associative Property)

where $a, b \in F$ and $x, y, z \in A$.

\section{Proposition 2.1. Cancellation Laws}

Let $(\mathrm{A}, \oplus, *, \bullet)$ be a region over the field $(\mathrm{F},+,$.$) .$ Since $(\mathrm{A}, \oplus, *)$ is a field, the following cancellation laws hold good in a region $(\mathrm{A}, \oplus, *, \bullet)$ by virtue of inheritance (proved in [16]):

(1) If $x \oplus y=x \oplus z$, then $y=z$ where $x, y, z \in A$.

(2) If $x \oplus y=z \oplus y$, then $x=z$ where $x, y, z \in A$.

(3) If $\mathrm{x} * \mathrm{y}=\mathrm{x} * \mathrm{z}$ where $\mathrm{x} \neq 0_{\mathrm{A}}$, then $\mathrm{y}=\mathrm{z}$ where $\mathrm{x}, \mathrm{y}, \mathrm{z}$ $\in \mathrm{A}$.

(4) If $\mathrm{x} * \mathrm{y}=\mathrm{z} * \mathrm{y}$ where $\mathrm{y} \neq 0$, then $\mathrm{x}=\mathrm{z}$ where $\mathrm{x}, \mathrm{y}$, $\mathrm{z} \in \mathrm{A}$.

However, it can be easily shown that the following two cancellation laws too hold good in a region A: -

(5) If $\mathrm{a} \bullet \mathrm{x}=\mathrm{a} \bullet \mathrm{y}$ where $\mathrm{a} \neq 0_{\mathrm{F}}$, then $\mathrm{x}=\mathrm{y}$ where $\mathrm{x}, \mathrm{y}$ $\in \mathrm{A}$ and $\mathrm{a} \in \mathrm{F}$.

(6) If $\mathrm{a} \bullet \mathrm{x}=\mathrm{b} \bullet \mathrm{x}$ where $\mathrm{x} \neq 0_{\mathrm{A}}$, then $\mathrm{a}=\mathrm{b}$ where $\mathrm{x} \in \mathrm{A}$ and $\mathrm{a}, \mathrm{b} \in \mathrm{F}$.

Besides the above six, there are a number of kinds of cancellation operations valid in the region $(\mathrm{A}, \oplus, *, \bullet)$ over the field $(\mathrm{F},+,$.$) , few of which are quoted below: -$

If $x, y \in A$ and $a, b \in F$, then

(7) If (a.b) $\bullet x=(a . c) \bullet y$ where $a \neq 0$ F, then $b \bullet x=c \bullet y$.

$(8)(a \bullet x) /(a \bullet y)=x / y$, where $a \neq 0$ F and $y \neq 0$.

(9) $((a . b) \bullet x) /((a . c) \bullet y)=(b \bullet x) /(c \bullet y)$, where $a \neq 0$ F.

(10) $((a . c) \bullet x) /((b . c) \bullet y=(a \bullet x) /(b \bullet y)$, where $c \neq 0$ F.

$(11)((\mathrm{a} \bullet \mathrm{x}) * \mathrm{y}) /((\mathrm{b} \bullet \mathrm{x}) * \mathrm{z})=(\mathrm{a} \bullet \mathrm{y}) /(\mathrm{b} \bullet \mathrm{z})$, where $\mathrm{x}$ $\neq 0_{\mathrm{A}}$.

$(12)((\mathrm{a} \bullet \mathrm{x}) * \mathrm{y}) /\left((\mathrm{a} \bullet \mathrm{z})^{*} \mathrm{t}\right)=(\mathrm{x} * \mathrm{y}) /(\mathrm{z} * \mathrm{t})$, where a $\neq 0_{\mathrm{F}}$ 
The following propositions are proved in [16] to be valid in a region.

\section{Proposition 2.2.}

In a region $(\mathrm{A}, \oplus, *, \bullet)$ over the field $(\mathrm{F},+,$.$) , the$ following results are true (keeping in mind that division by $0_{\mathrm{A}}$ or $0_{\mathrm{F}}$ is not permissible): -

If $\mathrm{x}, \mathrm{y}, \mathrm{z}, \mathrm{t} \in \mathrm{A}$, and $\mathrm{a}, \mathrm{b}, \mathrm{c}, \mathrm{d} \in \mathrm{F}$, then

(i) $\frac{x}{y} * \frac{z}{t}=\frac{x * z}{y * t}$

(ii) $\frac{x \oplus y}{z}=\left(\frac{x}{z}\right) \oplus\left(\frac{y}{z}\right)$

(iii) $x^{2} \sim y^{2}=(x \oplus y) *(x \sim y)$

(iv) $\frac{x}{y} \oplus \frac{z}{t}=\frac{x * t \oplus y * z}{y * t}$,

$$
\frac{a \bullet x \oplus b \bullet y}{c \bullet z}=\left(\frac{a \bullet x}{c \bullet z}\right) \oplus\left(\frac{b \bullet y}{c \bullet z}\right)
$$

(v)

$$
=\left(\frac{a}{c}\right) \bullet\left(\frac{x}{y}\right) \oplus\left(\frac{b}{c}\right) \bullet\left(\frac{y}{z}\right)
$$

\section{Proposition 2.3. Cross Multiplication Property}

In a region $\left(\mathrm{A}, \oplus,{ }^{*}, \bullet\right)$ over the field $(\mathrm{F},+,$.$) , the 'Cross$ Multiplication Property' is well valid.

i.e. If $\frac{x}{y}=\frac{z}{t}$, then $\mathrm{x} * \mathrm{t}=\mathrm{y} * \mathrm{z}$ and conversely, where $\mathrm{x}, \mathrm{y}, \mathrm{z}, \mathrm{t} \in \mathrm{A}$ and $\mathrm{y} \neq 0_{\mathrm{A}} \neq \mathrm{t}$.

This property is not necessarily valid in a division algebra as shown in Case-4 of subsection 2.1.

\section{Proposition 2.4. Componendo \& Dividendo Rule}

In a region $(\mathrm{A}, \oplus, *, \bullet)$ over the field $(\mathrm{F},+,$.$) , the$ following 'Componendo \& Dividendo Rule' is well valid:

(i) If $\frac{x}{y}=\frac{z}{t}$, then $\frac{x}{y}=\frac{z}{t}=\frac{x \oplus z}{y \oplus t}=\frac{x \sim z}{y \sim t}$, where $\mathrm{x}, \mathrm{y}, \mathrm{z}$, $\mathrm{t} \in \mathrm{A}$, and denominator $\neq 0_{\mathrm{A}}$.

(ii)

$$
\text { If } \quad \frac{x}{y}=\frac{z}{t}
$$

then

$\frac{x}{y}=\frac{z}{t}=\frac{(a \bullet x) \oplus(b \bullet z)}{(a \bullet y) \oplus(b \bullet t)}=\frac{a \bullet x \sim b \bullet z}{a \bullet y \sim b \bullet t}$, where $\mathrm{x}, \mathrm{y}, \mathrm{z}, \mathrm{t} \in$

$\mathrm{A}$, and denominator $\neq 0_{\mathrm{A}}$.

$$
\text { (iii) }(a \bullet x \oplus b \bullet y)^{n}=\sum_{r=0}^{n}\left(\left(\begin{array}{l}
n \\
r
\end{array}\right) \cdot a^{n-r} \cdot b^{r}\right) \bullet\left(x^{n-r} * y^{r}\right)
$$

\section{Birth of Compound Numbers}

In this section we introduce the concept of an important object called by imaginary object of a region. In fact we introduce now only the 'existence', not the 'identity', of an imaginary object of a region. We also introduce the concept of compound object and we then explore the birth of 'compound numbers'.

\section{Definition 3.1. Real Object of a Region.}

For a region $\mathrm{A}$, any member of the set $\mathrm{A}$ is called a "real object" of the region A.

\section{1. 'Existence' of Imaginary Objects of a Region.}

Consider a region $\mathrm{A}$, and let $\mathrm{E}_{1}(\mathrm{x})$ and $\mathrm{E}_{2}(\mathrm{x})$ be two single variable expressions valid in A. (It may be noted that an expression is regarded to be valid in an algebraic system $\mathrm{A}$ if it can be computed with the valid operations in A).

If the equality (not identity) $\mathrm{E}_{1}(\mathrm{x})=\mathrm{E}_{2}(\mathrm{x})$ is not satisfied by any element of the region $\mathrm{A}$, then we say that A has at least one "imaginary object" (or im-object or immember as synonyms) of 'imaginary object') which satisfies this equality. The imaginary objects (if exist) of the region $\mathrm{A}$ are to be called by A-io or Aio (in short).

Let us imagine that $\mathrm{i}$ is an imaginary object of $\mathrm{A}$ coming out of the equality $\mathrm{E}_{1}(\mathrm{x})=\mathrm{E}_{2}(\mathrm{x})$. Then we must have $\mathrm{E}_{1}(\mathrm{i})=\mathrm{E}_{2}(\mathrm{i})=\mathrm{z}$ (say) where $\mathrm{z} \in \mathrm{A}$.

It may be noted here that by definition we can only realize about the existence of an imaginary object of a region $\mathrm{A}$, but we can not trace its identity immediately. Because, an imaginary object of a region $\mathrm{A}$ is not a member of $\mathrm{A}$, and at the same time it is fact that we know nothing beyond the boundary of the set $\mathrm{A}$ at this stage. It is an open problem to us for further study and research.

\section{Example 3.1.}

Consider the region RR. If we take $E_{1}(x)=x^{2}+1$ and $E_{2}(x)=2 x-1$, then we understand the existence of at least one imaginary object of RR.

\section{Example 3.2.}

In the simple trivial region $\left(Z_{2}, \oplus, . ..\right)$ where $Z_{2}=\{0$, $1\}, \oplus$ is the "addition modulus 2 " operation and. is the 'multiplication modulus 2' of real numbers, we see that if we take $E_{1}(x)=2 x+1$ and $E_{2}(x)=0$, then we observe that there exist at least one imaginary object for this region $Z_{2}$. However, if we take $E_{1}(x)=x^{2}+1$ and $E_{2}(x)=0$ then it does not help us to know the existence of any imaginary object of $Z_{2}$.

Consider the region RR. Take $\mathrm{E}_{1}(\mathrm{x})=\mathrm{x}^{2}+1$ and $\mathrm{E}_{2}(\mathrm{x})=$ 0 . Clearly, this shows that the $R R$ region does have at least one imaginary object. But, we are not sure here whether there exist only finite number or infinite number of imaginary objects for RR.

The 'imaginary objects' for the regions which are the sets of numbers is to be called by 'imaginary numbers'. To avoid confusion between the existing concept of imaginary numbers and our notion of 'imaginary numbers' introduced here, we will call our notion of 'imaginary numbers' by the term 'im-numbers' henceforth.

If there exist im-numbers for the set of real numbers $\mathrm{R}$ then we will call them by the term R-im or rim (in short). If there exist im-numbers for the set of complex numbers $\mathrm{C}$ then we will call them by the term $\mathrm{C}$-im or cim (in short).

\subsection{Compound Numbers and Compound Objects}

Take $f(x)=x^{2}+1$. There is no $x$ in $R$ which satisfies the equation $f(x)=0$. It indicates that there is at least one rim in R. It is in fact well known that $\mathrm{R}$ has one rim which is $\mathrm{i}$. At this moment we will not debate on the issue "How many distinct rims $\mathrm{R}$ does have?", unless we do further 
work on it in the context of region mathematics. As on the existing literatures, there is one and only one rim which is i.

Next consider the region $C$. Take $f(z)=|z|^{2}+1$. There is no $\mathrm{z}$ in $\mathrm{C}$ which satisfies the equation $\mathrm{f}(\mathrm{z})=0$. It indicates that there is at least one cim in C. Say e is one cim in C generated from the above equation $\mathrm{f}(\mathrm{z})=0$. It means that $\mathrm{e}$ is an im-member of $\mathrm{C}$ for which $|\mathrm{e}|^{2}+1=0$. It is to be noted that $\mathrm{i}$ is an im-member of the region $\mathrm{R}$, not of the region $C$; and similarly e is an imaginary member of the region $C$, not of the region $R$. Thus for $z_{1}$ and $z_{2}$ of $C$, if $e$ is one $\operatorname{cim}$ of $\mathrm{C}$ then the object $\mathrm{d}=\left(\mathrm{z}_{1}+\mathrm{e} \mathrm{z}_{2}\right)$ is a compound number. The complex number $z_{1}$ is called the 'complex part' of the compound number $d$ and the complex number $z_{2}$ is called the 'imaginary part' of the compound number $\mathrm{d}$.

In general, suppose that $R_{1}, R_{2}, R_{3}, \ldots ., R_{n}$ are $n$ number of regions. A region may or may not have immember. Even if a region $R_{i}$ has a im-member, we need to explore how many more im-members does $R_{i}$ have. If $e_{i}$ is a im-member of the region $R_{i}$ and if $a, b$ are real objects of $R_{i}$ then $\left(a+b e_{i}\right)$ is a compound object of the region $R_{i}$. For the region $\mathrm{C}$, its compound objects are called the compound numbers.

If $x, y$ are in $R$ then the equation $x^{2}+y^{2}+1=0$ is not satisfied by any $x, y$ of $R$ and thus there many solutions of this equation in the form of $x=x_{1}+i x_{2}, y=y_{1}+i y_{2}$ which are complex numbers with respect to the region $\mathrm{R}$. These solutions are members of $\mathrm{C} \times \mathrm{C}$. The equation $|\mathrm{z}|^{2}+$ $1=0$ is to be solved for $\mathrm{z}$ in $\mathrm{C}$ and no such $\mathrm{z}$ is available in $\mathrm{C}$, leading to the existence of a cim. Consequently, searching for $(\mathrm{x}, \mathrm{y})$ satisfying the equation $\mathrm{x}^{2}+\mathrm{y}^{2}+1=0$ and searching for $z$ satisfying the equation $|z|^{2}+1=0$ are not same problems. In the first case we search for real numbers $\mathrm{x}$ and $\mathrm{y}$ from the jurisdiction $\mathrm{R}$ whereas in the second case we search for a complex number $\mathrm{z}$ from the jurisdiction $\mathrm{C}$.

History says that after the discovery of the rim i, a new number system took shape which is the set $\mathrm{C}$ of complex numbers. It is to be philosophically viewed that the existing notion of 'complex numbers' is with respect to its base 'real numbers'. In this sense ' $5 \mathrm{i}$ ' is an imaginary number to the set $\mathrm{R}$, not to the set $\mathrm{C}$. To the set $\mathrm{C}$ the number ' $5 \mathrm{i}$ ' is a core family-member. It is to be clearly understood that the issue of 'imaginary' or 'complex' is an relative issue, but local.

Thus our history says that the set $\mathrm{R}$ and later the discovery of $i$ gave birth of the set $\mathrm{C}$. In an analogous way we claim that the set $\mathrm{C}$ and the discovery of the cim e (and other cims, if exist) will lead to the discovery of a new set of numbers. Let us call this set by the set of "Compound Numbers" denoted by E. Our immediate need is to discover the fundamental operations on $\mathrm{E}$ (like additions, multiplications, etc.) and then to study $\mathrm{E}$ as a possible algebra, and more..... It is obvious that $\mathrm{E}$ forms a group with respect to the binary operation ' + ' defined by: for the compound numbers $d_{1}=z_{11}+e z_{12}$ and $d_{2}=z_{21}+e z_{22}, d_{1}$ $+\mathrm{d}_{2}=\left(\mathrm{z}_{11}+\mathrm{z}_{21}\right)+\mathrm{e}\left(\mathrm{z}_{12}+\mathrm{z}_{22}\right)$.

In the next section we introduce two parts of region mathematics. In the first part we introduce that for every complete region A, there is a corresponding "Theory of Numbers". We then show that the existing classical 'theory of numbers' is a particular instance of our newly introduced notion of "Theory of Numbers". In the second part we introduce another new field "Object Geometry" corresponding to a complete region $\mathrm{A}$, and also we show that the existing classical 'geometry' is a particular instance of our newly introduced "object geometry".

\section{On "Theory of Numbers" of a Complete Region A}

First of all let us recall and review few definitions from $[16,17]$. Consider any real region $\mathrm{A}=(\mathrm{A}, \oplus, *, \bullet)$ over the field $(\mathrm{R},+,$.$) . Then \mathrm{A}$ forms a Calculus Space if the following conditions are true:

(i) $\mathrm{A}$ is an extended real region.

(ii) $\mathrm{A}$ is a normed complete metric space with respect to a norm $\|$.$\| and the corresponding induced metric \rho$ (x, y) $=\|\mathrm{x} \sim \mathrm{y}\|$, (i.e. $\|\mathrm{x}\|=\rho\left(\mathrm{x}, 0_{\mathrm{A}}\right)$ ).

(iii) The norm $\|\cdot\|$ is 2-to-1 bijective mapping from $\mathrm{A}$ $-\left\{0_{\mathrm{A}}\right\}$ to $\mathrm{R}^{+}$.

(iii) $\mathrm{A}$ is a chain w.r.t. the total order relation $\leq$.

If we choose the real region $A$ to be the $R R$ region and $\|\mathrm{x}\|=|\mathrm{x}|$ in RR where $\rho(\mathrm{x}, \mathrm{y})=\|\mathrm{x}-\mathrm{y}\|=|\mathrm{x}-\mathrm{y}|$ and the $\mathrm{RR}$ region is a chain w.r.t. the crisp order relation " $\leq$ ", then the corresponding calculus happens to be the classical calculus (developed independently by Newton and Leibniz).

The following fact may be recalled that the metric associated with this norm i.e. the metric $\rho(\mathrm{x}, \mathrm{y})=\|\mathrm{x} \sim \mathrm{y}\|$ has the following special properties:

(i) 'translation invariance', i.e. $\forall \mathrm{z} \in \mathrm{A}$ we have $\rho$ (x $\oplus \mathrm{z}, \mathrm{y} \oplus \mathrm{z})=\rho(\mathrm{x}, \mathrm{y})=\|\mathrm{x} \sim \mathrm{y}\|$, and

(ii) 'homogeniety', i.e. $\forall \mathrm{r} \in \mathrm{F}$ we have $\rho(\mathrm{r} \bullet \mathrm{x}, \mathrm{r} \bullet \mathrm{y})$ $=|\mathrm{r}| \cdot\|\mathrm{x} \sim \mathrm{y}\|=|\mathrm{r}| \cdot \rho(\mathrm{x}, \mathrm{y})$.

We defined 'Absolute Partition' of the region $\mathrm{A}=(\mathrm{A}$, $\left.\oplus,{ }^{*}, \bullet\right)$ in [17]. Consider a partition $\mathrm{P}_{\mathrm{A}}$ of a region $\mathrm{A}$ (forming calculus space) into three mutually disjoint nonnull sets $\mathrm{A}^{+}, \mathrm{A}^{-}$and $\left\{0_{\mathrm{A}}\right\}$ such that

(i) $\mathrm{A}^{+}=\left\{\mathrm{a}: \mathrm{a} \in \mathrm{A}\right.$ and $\left.0_{\mathrm{A}}<\mathrm{a}\right\}$

(ii) $\mathrm{A}^{-}=\left\{\mathrm{a}: \mathrm{a} \in \mathrm{A}\right.$ and $\left.\mathrm{a}<0_{\mathrm{A}}\right\}$.

Clearly, $\forall \mathrm{a} \in \mathrm{A}^{+}, \sim \mathrm{a} \in \mathrm{A}^{-}$and $\forall \mathrm{b} \in \mathrm{A}^{-}, \sim \mathrm{b} \in \mathrm{A}^{+}$.

(Note: we say that $\mathrm{a}<\mathrm{b}$ iff $\mathrm{a} \leq \mathrm{b}$ and $\mathrm{a} \neq \mathrm{b}$ ).

This partition $\mathrm{P}_{\mathrm{A}}$, once made, must be regarded as an 'absolute partition' for the region A over which one desires to develop a calculus and any branch of region mathematics in any direction. It is called to be absolute in the sense that it generates the sign of every object of A, positive or negative, which will remain absolute for the complete literature of the corresponding calculus or corresponding region mathematics. The elements of $\mathrm{A}^{+}$are said to be positive objects and the elements of $\mathrm{A}^{-}$are said to be negative objects. The object $0_{\mathrm{A}}$ is neither in $\mathrm{A}^{+}$nor in $\mathrm{A}^{-}$, and so we say that $0_{\mathrm{A}}$ is neither a positive object nor a negative object. The attribute of being positive or negative is called the sign of the object, and $0_{\mathrm{A}}$ is not considered to have a sign.

A real region which forms a calculus space satisfying the above properties is called a "complete region". For instance, the region RR is a complete region. For a given complete region $[16,17]$, a line can be drawn with positive objects to the right, and negative objects to the left of $0_{\mathrm{A}}$. Thus the 'positive direction' of $\mathrm{X}$-axis and the 'negative direction' of $\mathrm{X}$-axis can be well understood and the line 
which the objects of the region A is considered to lie upon is called the Object Line (see Figure 1 and Figure 2).

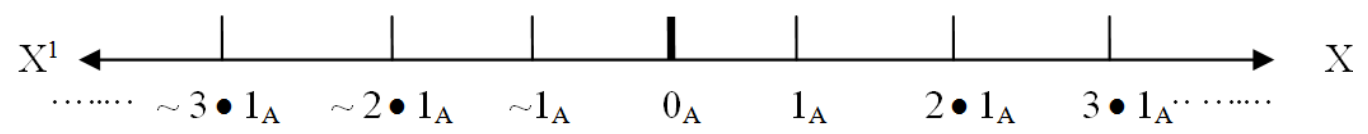

Figure 1. Object line of the region A with consecutive equi-spaced object points.

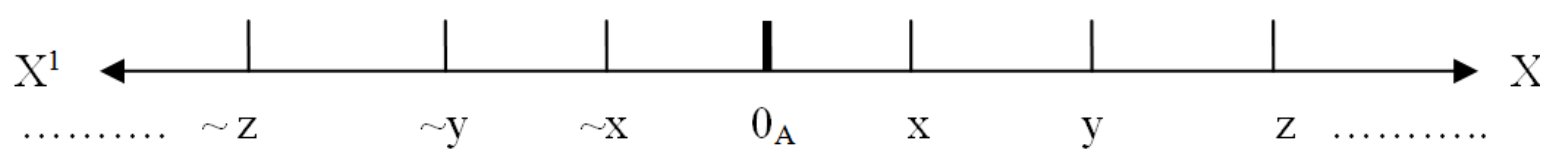

Figure 2. Objects line of the region A, a general view

The term 'equi-spaced' in the caption of Figure 1 is well understood in the sense of the corresponding metric (or norm) of the region A. Since $\mathrm{A}=\left(\mathrm{A}, \oplus,,^{*}, \bullet\right)$ is complete (normed complete metric space), there are no "points missing" from it (inside or at the boundary). Since $\mathrm{A}$ is a chain, every object has a unique address on this linear continuum X.

In the 'Theory of Objects' introduced in [16] we defined composite objects, prime objects, real objects, imaginary objects etc. and then explained how the classical notion of composite numbers, prime numbers, real numbers, imaginary numbers etc. of the classical 'Theory of Numbers' are special instances in the 'Theory of Objects' out of a particular region RR (corresponding to the set of real numbers $\mathrm{R}$ and the set of Complex numbers). In the next section we define the notion of object integer and consequently we introduce an important new field called by "Theory of Numbers" for a complete region. The proposed "Theory of Numbers" is not developed here as an extension of the classical 'Theory of Numbers'. Rather it has happened that the classical 'Theory of Numbers' is just a special case of our proposed "Theory of Numbers". The identical title will not create any confusion once the subject develops further in the next section here.

\subsection{Unit Length \& Inverse Unit Length in a complete region $A$}

Consider a complete region $\mathrm{A}=(\mathrm{A}, \oplus, *, \bullet)$. For $\mathrm{x}_{\mathrm{A}} \in$ $A$, we use the notation $x_{a}$ to denote $\left\|x_{A}\right\|=x_{a}$ which is a positive real number. If $x_{A}$ is a positive object on the object line, then the distance of $\mathrm{x}_{\mathrm{A}}$ from the point $\mathrm{O}$ is denoted by $\mathrm{x}_{\mathrm{a}}$ which is a positive real number (we use the convention that $\sim \mathrm{X}_{\mathrm{A}}$ is at a distance of $-\mathrm{x}_{\mathrm{a}}$ from the point $\mathrm{O})$. Corresponding to the unit element $1_{\mathrm{A}}$ of the complete region $\mathrm{A}$, the positive real number $1_{\mathrm{a}}$ (i.e. $\left\|1_{\mathrm{A}}\right\|$ ) is called the 'unit length' in A. Clearly $0_{a}=0$, and it may also be noted here that in general $1_{\mathrm{a}} \neq 1$ (where 0 is the $\mathrm{o}_{\mathrm{RR}}$ and 1 is the $1_{\mathrm{RR}}$ ). Suppose that $1 / 1_{\mathrm{a}}=\partial_{\mathrm{a}}$. The positive real number $\partial_{\mathrm{a}}$ is called 'Inverse Unit Length' in A. Clearly $1_{\mathrm{a}}$. $\partial_{\mathrm{a}}=1$ for $\mathrm{A}$, and for the particular complete region RR we have $1_{\mathrm{rr}}=\partial_{\mathrm{rr}}=1\left(=1_{\mathrm{RR}}\right)$.

\section{2. 'Ontegers' in the Complete Region A}

Consider the object $\mathrm{x}_{\mathrm{A}}$ in the complete region $\mathrm{A}$. Consider the real number $\mathrm{x}_{\mathrm{a}} / 1_{\mathrm{a}}$ i.e. $\mathrm{x}_{\mathrm{a}} \cdot \partial_{\mathrm{a}}$ which let us denote by the symbol $x$. Thus $x=x_{a} / 1_{a}=x_{a} \cdot \partial_{a}$, which means $\mathrm{x}_{\mathrm{a}}=\mathrm{x} .1_{\mathrm{a}} \forall \mathrm{x}_{\mathrm{A}} \in \mathrm{A}$. It may be noted here that in general as $1_{\mathrm{a}} \neq 1$, in a similar way $\mathrm{x}_{\mathrm{a}} \neq \mathrm{x}$. However for a particular instance of the complete region RR, we have $x_{\text {rr }}$ $=\mathrm{x}\left(=\mathrm{x}_{\mathrm{RR}}\right)$.

If $m$ is a real integer, then the object $m_{A}$ is called an 'object integer' or 'onteger' in the complete region A. Thus the ontegers in $\mathrm{A}$ are $0_{\mathrm{A}}, \oplus 1_{\mathrm{A}}, \sim 1_{\mathrm{A}}, \oplus 2_{\mathrm{A}}, \sim 2_{\mathrm{A}}, \oplus$ $3_{\mathrm{A}}, \sim 3_{\mathrm{A}}, \ldots$. etc. The ontegers $\oplus 1_{\mathrm{A}}, \oplus 2_{\mathrm{A}}, \oplus 3_{\mathrm{A}}, \oplus$ $4_{\mathrm{A}}, \ldots \ldots .$. etc. are 'positive ontegers' and the ontegers $\sim 1_{\mathrm{A}}$, $\sim 2_{\mathrm{A}}, \sim 3_{\mathrm{A}}, \sim 4_{\mathrm{A}}, \ldots \ldots .$. etc. are 'negative ontegers' in the complete region A. It is to be carefully noted that corresponding to any onteger $\oplus \mathrm{m}_{\mathrm{A}}$ of the complete region $\mathrm{A}$, the distance $\mathrm{m}_{\mathrm{a}}$ from the point $\mathrm{o}_{\mathrm{A}}$ on the object line need not necessarily be a real integer (as defined in classical traditional sense), and similarly corresponding to any onteger $\sim \mathrm{m}_{\mathrm{A}}$, the distance $-\mathrm{m}_{\mathrm{a}}$ need not necessarily be a real integer (as defined in classical traditional sense). If we imagine a common object line for different complete regions $\mathrm{RR}, \mathrm{A}, \mathrm{B}, \mathrm{C}, \mathrm{D}, \ldots .$. etc. with the zero element 0 , $0_{\mathrm{A}}, 0_{\mathrm{B}}, 0_{\mathrm{C}}, 0_{\mathrm{D}}, \ldots .$. respectively, being situated at the exactly same point on the object line, then it is obvious that the respective unit elements $1,1_{\mathrm{A}}, 1_{\mathrm{B}}, 1_{\mathrm{C}}, 1_{\mathrm{D}}, \ldots$ etc. will be situated in general at different points on the common line because of the fact that the 'unit length' is region dependent. Thus, in general the points $\mathrm{x}, \mathrm{x}_{\mathrm{A}}, \mathrm{x}_{\mathrm{B}}, \mathrm{x}_{\mathrm{C}}$, $\mathrm{x}_{\mathrm{D}}, \ldots$ etc. will be situated at different locations on the common object line. Distance (if measured in a common scale, say with the help of real numbers) between two consecutive ontegers for any given complete region A on the object line will be same, but will be different for different complete regions. On the RR region line i.e. on the real number line, distance of the object $\oplus 1_{\mathrm{RR}}$ or $\sim 1_{\mathrm{RR}}$ from the object $0_{\mathrm{RR}}$ (i.e. distance of the real number +1 or -1 from the number 0 ) is of unit length called us by 'one'. It may be noted that for every $\mathrm{x}_{\mathrm{A}} \in \mathrm{A}, \mathrm{x}_{\mathrm{a}}$ is in R. It may happen that the real number 1 of $R$ (i.e. the object $1_{R R}$ of $\mathrm{RR}$ ) is not an onteger (integer) in the "Theory of numbers" of the region $\mathrm{A}$ and the real number $1_{\mathrm{a}}$ of the region $\mathrm{A}$ is not an integer in the classical "Theory of numbers" i.e. in the "Theory of numbers" of the region RR. The main source of difference lies in the difference of size of 'unit length' of different complete regions. This important result unearths the fact that every complete region has its own "Theory of Numbers". Thus "Theory of Numbers" is different for different complete regions, whereas the classical "Theory of Numbers" being practiced by us traditionally so far is just the same of a particular complete region which is $R R$. The following proposition is straightforward and quite important.

\section{Proposition 4.1.}


Corresponding to a real number $\mathrm{x}(-\mathrm{x})$, there is a unique object $\oplus \mathrm{x}_{\mathrm{A}}\left(\sim \mathrm{x}_{\mathrm{A}}\right)$ in every complete region $\mathrm{A}$ and hence a unique corresponding real number $\mathrm{x}_{\mathrm{a}}\left(-\mathrm{x}_{\mathrm{a}}\right)$.

\section{Definition 4.1. ' $R_{A}$ value' of a real number $x$ in $A$}

Let $A$ be a complete region. Consider the 1-to-1 mapping $\mathrm{R}_{\mathrm{A}}: \mathrm{R} \rightarrow \mathrm{R}$ defined by $\mathrm{R}_{\mathrm{A}}(\mathrm{x})=\mathrm{x}_{\mathrm{a}} \forall \mathrm{x} \in \mathrm{R}$. Then the real number $x_{a}$ is called the ' $R_{A}$ value' of $x$ denoted by $\mathrm{R}_{\mathrm{A}}(\mathrm{x})=\mathrm{x}_{\mathrm{a}}$ in the complete region $\mathrm{A}$. Clearly, in that case $\mathrm{R}_{\mathrm{A}}(-\mathrm{x})=-\mathrm{x}_{\mathrm{a}}$. Also $\mathrm{R}_{\mathrm{A}}(0)=0_{\mathrm{a}}$, and $\mathrm{R}_{\mathrm{A}}(1)=1_{\mathrm{a}}$. It is obvious that $R_{R R:} R \rightarrow R$ is an identity mapping.

\section{Definition 4.2. 'Set of $R$ values'}

The collection of all complete regions is called the region universe $\Sigma$. If RR, A, B, C, D, .... are the complete regions in the region universe $\Sigma$, then for any given real number $\mathrm{x}$ the set $\Sigma_{\mathrm{x}}=\left\{\mathrm{x}, \mathrm{x}_{\mathrm{a}}, \mathrm{x}_{\mathrm{b}}, \mathrm{x}_{\mathrm{c}}, \mathrm{x}_{\mathrm{d}}, \ldots\right\}$ is called the 'Set of R values' of $\mathrm{x}$ in the region universe $\Sigma$. Although we call $\Sigma_{\mathrm{x}}$ a set, it could be a multiset (bag) too. Collection of $\mathrm{R}$ values of the real number 1 is the set (multiset) of all unit length values forming $\Sigma_{1}$, and the Collection of $\mathrm{R}$ values of the real number 0 is the set (multiset) $\Sigma_{0}$.

\section{Definition 4.3. Natural Numbers of a complete region}

Consider a complete region $\mathrm{A}$. The real numbers $\oplus 1_{\mathrm{a}}$, $\oplus 2_{\mathrm{a}}, \oplus 3_{\mathrm{a}}, \oplus 4_{\mathrm{a}}, \ldots . .$. .. are called the Natural Numbers of A. For instance, for the complete region RR, the natural numbers are $1_{\mathrm{rr}}, 2_{\mathrm{rr}}, 3_{\mathrm{rr}}, 4_{\mathrm{rr}}, \ldots \ldots .$. , i.e. $1,2,3,4, \ldots$. etc. which are in this particular case $1_{\mathrm{RR}}, 2_{\mathrm{RR}}, 3_{\mathrm{rRR}}, 4_{\mathrm{RR}}, \ldots \ldots \ldots$, etc.

Once the notion of "Theory of Numbers" of a complete region $\mathrm{A}$ is developed, we are now in a position to initiate a corresponding geometry.

\section{5. 'Object Geometry' of a Complete Region A}

For developing a new geometry called by "Object Geometry", be it in a two dimensional object coordinate system, or in an n-dimensional object coordinate system, at least one complete region $\mathrm{A}=\left(\mathrm{A}, \oplus,{ }^{*}, \bullet\right)$ is required. Consider the object line and the corresponding $\mathrm{X}$-axis for A. Consider a point $\mathrm{x}_{\mathrm{A}}$ on the $\mathrm{X}$-axis. Then for the infinitesimal small positive object $\Delta \mathrm{x}_{\mathrm{A}}$, the point $\left(\mathrm{x}_{\mathrm{A}}+\right.$ $\left.\Delta \mathrm{x}_{\mathrm{A}}\right)$ will be at a distance $\left\|\Delta \mathrm{x}_{\mathrm{A}}\right\|$ from the point $\mathrm{x}_{\mathrm{A}}$ along the positive direction of $\mathrm{X}$-axis and the point $\left(\mathrm{x}_{\mathrm{A}}-\Delta \mathrm{x}_{\mathrm{A}}\right)$ will be at a distance $\left\|\Delta \mathrm{x}_{\mathrm{A}}\right\|$ from the point $\mathrm{x}_{\mathrm{A}}$ along the negative direction of $\mathrm{X}$-axis.

\subsection{The Coordinate Plane of Complete Region $A=(A, \oplus, *, \bullet)$}

We introduce first of all 2-D object geometry. It is a system of geometry where the position of points on the plane is described using an ordered pair of objects. A plane is a flat surface that goes on forever in both directions. If we were to place a point on the plane, object coordinate geometry gives us a way to describe exactly where it is by using two objects. Points are placed on the "object coordinate plane" as shown below in Figure 3. It has two scales - one running across the plane called the " $\mathrm{x}_{\mathrm{A}}$-axis" and another at right angles to it called the " $\mathrm{y}_{\mathrm{A}}$ - axis". The point where the two axes cross is called the origin at which both $\mathrm{x}_{\mathrm{A}}$ and $\mathrm{y}_{\mathrm{A}}$ are $0_{\mathrm{A}}$. On the $\mathrm{x}_{\mathrm{A}}$-axis, as explained earlier that objects to the right of origin are positive and those to the left are negative. On the $\mathrm{y}_{\mathrm{A}}$-axis, objects above the origin are positive and those below are negative.

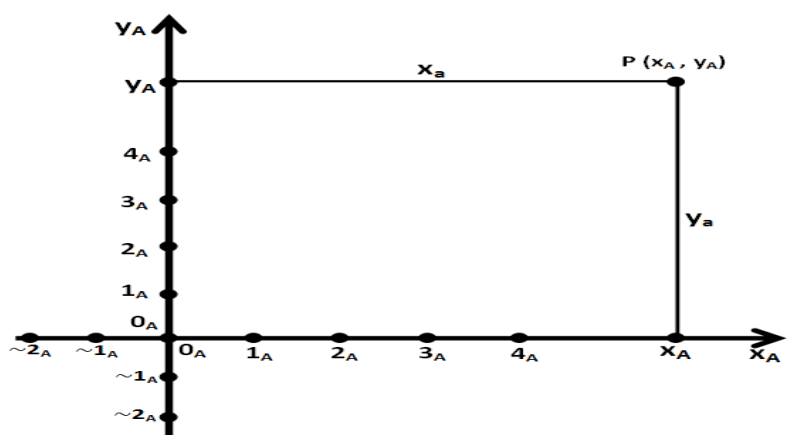

Figure 3. Objects coordinates on object plane of the region A

A point's location on the plane is given by two objects in the form of object coordinates $\left(\mathrm{x}_{\mathrm{A}}, \mathrm{y}_{\mathrm{A}}\right)$, the first coordinate reveals where it is away from the $\mathrm{y}_{\mathrm{A}}$-axis at parallel to the $\mathrm{x}_{\mathrm{A}}$-axis and the second coordinate reveals where it is away from the $\mathrm{x}_{\mathrm{A}}$-axis at parallel to the $\mathrm{y}_{\mathrm{A}}$-axis (see Figure 3 above). There are four quadrants and sign convention rule is same as that of classical coordinate geometry, i.e. same for all the object geometry of all the complete regions.

\subsection{Slope of an Object Line on the Object Plane}

Slope of an object line passing through the two object points $\mathrm{P}\left(\mathrm{x}_{2 \mathrm{~A}}, \mathrm{y}_{2 \mathrm{a}}\right)$ and $\mathrm{Q}\left(\mathrm{x}_{1 \mathrm{~A}}, \mathrm{y}_{1 \mathrm{a}}\right)$ is the real number $\mathrm{m}_{\mathrm{a}}$ given by (as shown in Figure 4$)$ :

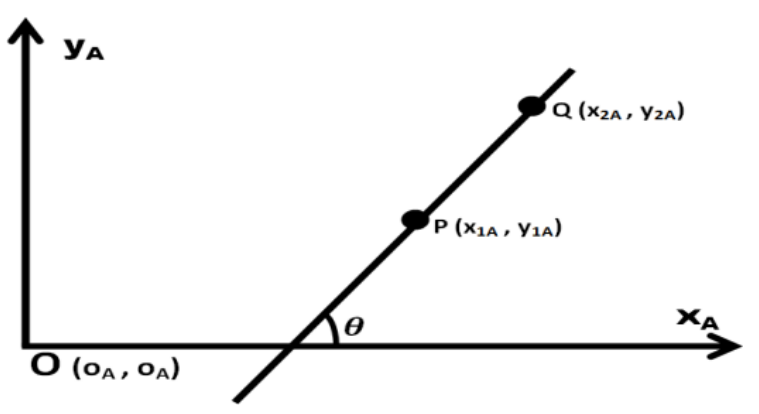

Figure 4. Slope of an objects line.

$$
\begin{aligned}
& m_{a}=\tan \theta=\left(y_{2 a}-y_{1 a}\right) /\left(x_{2 a}-x_{1 a}\right) \\
& =\left(y_{2} \cdot 1_{a}-y_{1} \cdot 1_{a}\right) /\left(x_{2} \cdot 1_{a}-x_{1} \cdot 1_{a}\right) \\
& =\left(y_{2}-y_{1}\right) /\left(x_{2}-x_{1}\right)
\end{aligned}
$$

This implies that slope of a line does not depend on the 'unit length' of the region. it is an absolute quantity irrespective of the region on which the object plane is drawn. Thus, slope of a line is region independent.

Proposition 5.1 Pythagorous Theorem is valid in every Object Geometry.

Proof: Let PQR be a right angled triangle (the angle PQR being the right angle) on the object plane of a complete region A (Figure 5(a)). 

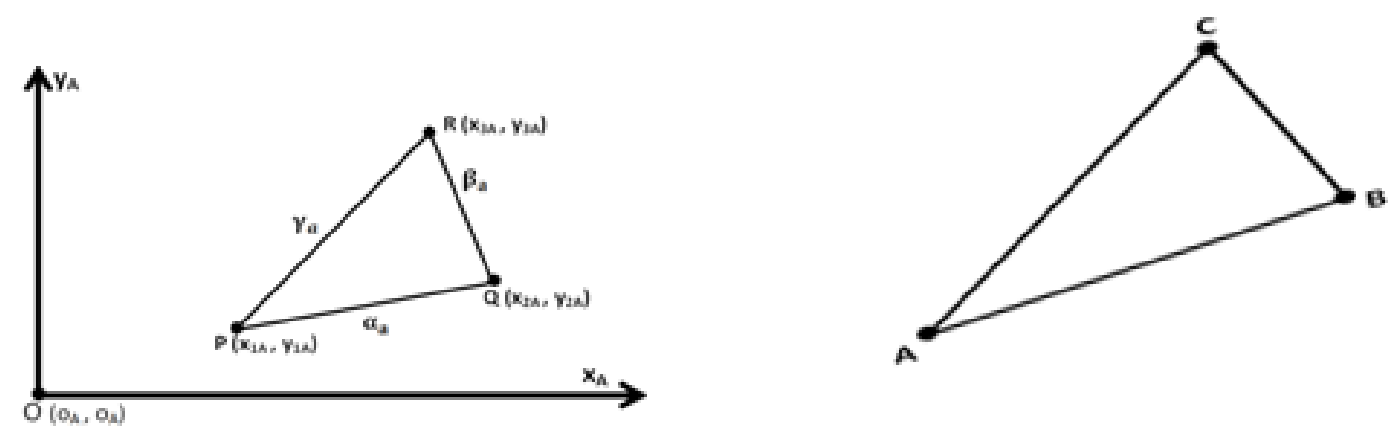

Figure 5. (a), (b). Right angled triangles in two object planes

Now, using the homogeneity property of the metric $\rho$ $(\mathrm{x}, \mathrm{y})=\|\mathrm{x} \sim \mathrm{y}\|$, we can find a right-angled triangle $\mathrm{ABC}$ (in fact there are infinite numbers of such triangles) on the real coordinate plane i.e. on the object plane of $\mathrm{RR}$ region, where

$$
\frac{P Q}{A B}=\frac{Q R}{B C}=\frac{P R}{A C}=1_{a}
$$

Since slope of a line is region independent, the rightangled property of the classical triangle $\mathrm{ABC}$ is guaranteed (the angle $\mathrm{ABC}$ being the right angle, see Figure 5 (b)) on the coordinate plane from the right-angled property of the triangle PQR. Since Pytharogous theorem is valid in the triangle $\mathrm{ABC}$, it is also so in the triangle PQR using equation (1). Hence proved.

\subsection{Distance between two Object Points on the Object Plane}

Consider the two object points $\mathrm{P}\left(\mathrm{x}_{2 \mathrm{~A}}, \mathrm{y}_{2 \mathrm{a}}\right)$ and $\mathrm{Q}\left(\mathrm{x}_{1 \mathrm{~A}}, \mathrm{y}_{1 \mathrm{a}}\right)$ on the object plane (see Figure 6). Distance PQ is the positive real number $r_{a}$ where

$$
r_{a}=\left\{\left(y_{2 a}-y_{1 a}\right)^{2}+\left(x_{2 a}-x_{1 a}\right)^{2}\right\}^{1 / 2}
$$

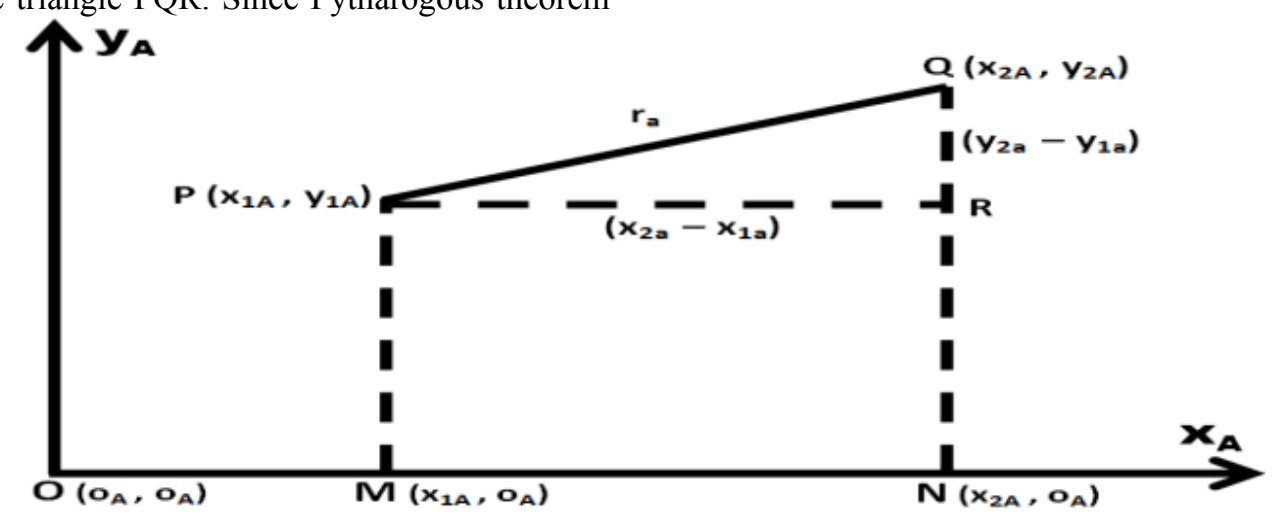

Figure 6. Distance between two object points

\subsection{Equation of a Line}

Equation of an object line whose slope is $m_{a}$ is

$$
y_{a}=m_{a} \cdot x_{a}+c_{a}
$$

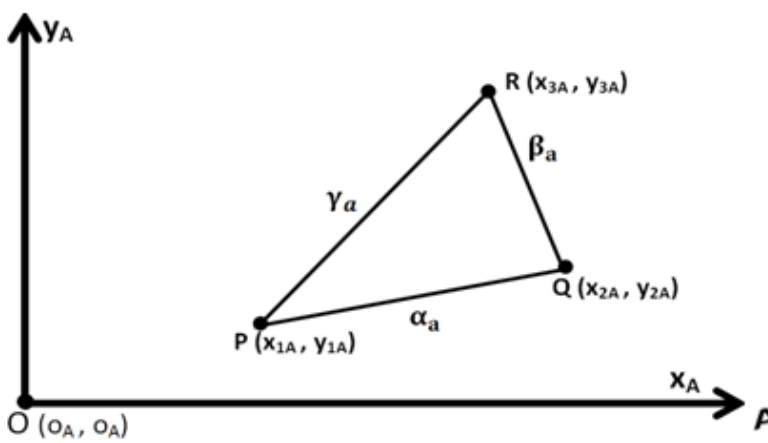

Figure 7. An object line having intercept $c_{a}$ on $y_{A}$ axis
Equation of an object line having slope $\mathrm{m}_{\mathrm{a}}$ and passing through the object point $\mathrm{Q}\left(\mathrm{x}_{1 \mathrm{~A}}, \mathrm{y}_{1 \mathrm{a}}\right)$ is $\left(\mathrm{y}_{\mathrm{a}}-\mathrm{y}_{1 \mathrm{a}}\right)=\mathrm{m}_{\mathrm{a}} \cdot\left(\mathrm{x}_{\mathrm{a}}-\right.$ $\mathrm{x}_{1 \mathrm{a}}$ ).

Equation of an object line passing through the two object points $\mathrm{P}\left(\mathrm{x}_{2 \mathrm{~A}}, \mathrm{y}_{2 \mathrm{a}}\right)$ and $\mathrm{Q}\left(\mathrm{x}_{1 \mathrm{~A}}, \mathrm{y}_{1 \mathrm{a}}\right)$ is $\left(\mathrm{y}_{\mathrm{a}}-\mathrm{y}_{1 \mathrm{a}}\right)=\mathrm{m}_{\mathrm{a}}$. $\left(\mathrm{x}_{\mathrm{a}}-\mathrm{x}_{1 \mathrm{a}}\right)$, where $\mathrm{m}_{\mathrm{a}}=\left(\mathrm{y}_{2 \mathrm{a}}-\mathrm{y}_{1 \mathrm{a}}\right) /\left(\mathrm{x}_{2 \mathrm{a}}-\mathrm{x}_{1 \mathrm{a}}\right)$.

\subsection{Object Circle on the Object Plane}

Equation of a Object Circle with centre at $\left(0_{\mathrm{A}}, 0_{\mathrm{A}}\right)$ and radius $r_{a}$ is $x_{a}{ }^{2}+y_{a}{ }^{2}=r_{a}{ }^{2}$ or, $x^{2}+y^{2}=r^{2}($ in the region $A)$.

Note that $x^{2}+y^{2}=25$ is the equation of an object circle on the object plane of the region $\mathrm{A}$, and hence this 
equation is not region-independent. The radius of this circle is $5_{\mathrm{a}}$ and centre is at $\left(0_{\mathrm{A}}, 0_{\mathrm{A}}\right)$.

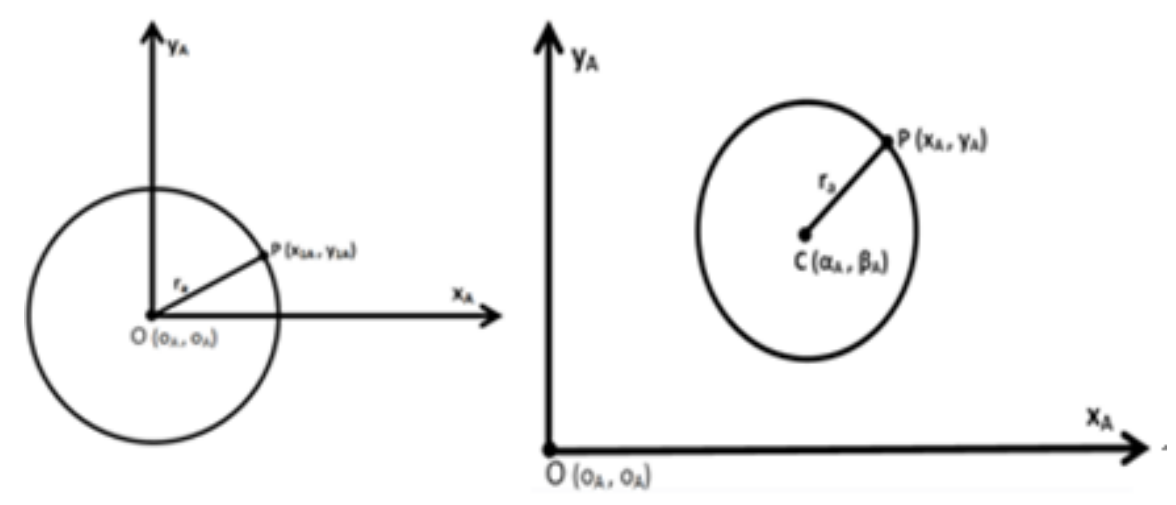

Figure 8 (a), (b). Objects circles

If $1_{a}>1$, then the object circle $x^{2}+y^{2}=r^{2}$ is a bigger circle than the classical circle $x^{2}+y^{2}=r^{2}$; if $1_{a}<1$, then the object circle $x^{2}+y^{2}=r^{2}$ is a smaller circle than the classical circle $x^{2}+y^{2}=r^{2}$; and if $1_{a}=1$, then the object circle $x^{2}+y^{2}=r^{2}$ is of same size with the classical circle $\mathrm{x}^{2}+\mathrm{y}^{2}=\mathrm{r}^{2}$.

Equation of a Object Circle with centre at $\left(\alpha_{\mathrm{A}}, \beta_{\mathrm{A}}\right)$ and radius $r_{a}$ is

$$
\begin{aligned}
& \left(y_{a}-\beta_{a}\right)^{2}+\left(x_{a}-\alpha_{a}\right)^{2}=r_{a}^{2} \\
& \text { or, }(y-\beta)^{2}+(x-\alpha)^{2}=r^{2}
\end{aligned}
$$

It is to be noted that the circle $(y-\beta)^{2}+(x-\alpha)^{2}=r^{2}$ on the object plane of region $A$ and the classical circle $(y-$ $\beta)^{2}+(x-\alpha)^{2}=r^{2}$ in 2-D classical coordinate geometry are two different circles having different centres and radii in general.

The classical geometry (2-D geometry, 3-D or higher dimensional geometry) being practiced by the world mathematicians so far at elementary or higher level is a particular case of the 'Object Geometry'. Other results of the classical geometry can be similarly studied and explored in object geometry which we will attempt in our future work.

\section{Conclusion}

The classical "Theory of Numbers" and "Geometry" developed so far is mainly based on the set $\mathrm{R}$ of real numbers, extended with two infinities (and then took its shape further with advanced higher mathematics). The growth of these two giant subjects at every stage so far required fluent applications of various operations and results which are valid in the set of real numbers. In many of the existing literatures, $\mathrm{R}$ is assumed to be just a field or a division algebra. But this assumption is not true (rather, let us say 'not sufficient') because of the fact that using the properties of a 'field' or a 'division algebra' or of any existing algebra other than region algebra [16], many of the formulas, rules, results or materials of elementary as well as higher algebra can not have the validity as shown with several examples in [16]. A careful study of the region algebra will clarify that many of the results, formulas, equalities, identities, rules, etc. of elementary algebra (say, the algebra practiced at high school level or higher level) are not valid in the fields or in division algebras or in any existing algebras in general, but in regions only. Fortunately the set $\mathrm{R}$ is a trivial example of real region called by region $R R$ in [16], and in a hidden way $\mathrm{R}$ has been providing the world mathematicians all the properties of region algebra, not just the properties of division algebra or any of the existing algebra being proven to be insufficient in the work [16]. Interestingly, the field $\mathrm{R}$ (or the division algebra $\mathrm{R}$ ) satisfies few additional properties trivially by which it qualifies to become a real region; and consequently the classical geometry never faced any computational constraints or invalidity even assuming $\mathrm{R}$ to be a field or division algebra or any of the existing algebra just. This is a major breakthrough, for a clear understanding of which one needs a serious study of the region algebra, even if apparently or initially the work [16] may appear to be an ordinary issue to the world mathematicians. In this paper we make further study of the "Theory of Objects", introduce two new theories called by "Theory of Numbers" and "Object Geometry". These two topics will grow a lot with time, the present work being just an initialization. We have identified 'What are the minimum properties which need to be satisfied by a set A so that a geometry can be developed over the platform A?'. It has been explained how the classical "Theory of Numbers" being practiced by the world so far happens to be a particular instance of our new "Theory of Numbers" of a complete region. For a non-example, the set of all triangular fuzzy numbers do not form a real region with respect to its commonly used operations, and hence can not open any platform to develop any calculus as mentioned in [17], can not open any Theory of Numbers or Geometry at the present form.

In this paper our work starts defining imaginary objects (if exist) of a region, compound objects (subject to existence of imaginary objects) of a region. As a particular case we study the imaginary number $i$ of the set $R$ of real numbers as an instance of imaginary object of a region, here it is called by rim. We then find imaginary object of the region $\mathrm{C}$ (set of complex numbers) which we call by the notation $\mathrm{cim}$ of $\mathrm{C}$. One cim we have extracted here which we name by $e$. If $x$ and $y$ are in $R$, then corresponding to the $\operatorname{rim} \mathrm{i}$ of $\mathrm{R}$ the object $(\mathrm{x}+\mathrm{iy})$ is $\mathrm{a}$ complex number for the set $\mathrm{R}$ of real numbers. if $\mathrm{e}$ is one $\operatorname{cim}$ of $C$ then the object $\left(z_{1}+e z_{2}\right)$ is a compound number. Analogously, if $z_{1}$ and $z_{2}$ are in $C$ then corresponding to the cim e of $C$ the object $\left(z_{1}+e z_{2}\right)$ is a compound number for the set $\mathrm{C}$ of complex numbers. The rim $\mathrm{i}$ is imaginary for $\mathrm{R}$, not for $\mathrm{C}$; and rim is a core member of $\mathrm{C}$ not of $\mathrm{R}$. 
Thus rim is a real object of $\mathrm{C}$. The cim e is imaginary for $\mathrm{C}$, not for any other region. The cim e is not a member of $C$, i.e. not a real object of C. In this paper we have seen the birth of a new type of numbers called by 'compound numbers'. The set of all compound numbers is denoted by E. At this moment we need to identify E precisely, by identifying precisely its members, characteristic properties, results, etc. which will be our future research work. Subsequently in due time, we need to revisit many of the long standing existing results (see $[1-15,18,19]$ ), viz:

(i) R, C, H, O are the only normed division algebras.

(ii) the only associative real division algebras are real numbers, complex numbers, and quaternions.

(iii) The Cayley algebra is the only non-associative division algebra.

(iv) The algebras of real numbers, complex numbers, quaternions, and Cayley numbers are the only ones where multiplication by unit "vectors" is distance-preserving.

\section{References}

[1] Alperin, J. L., with R. B. Bell, Groups and Representations, Graduate Texts in Mathematics, Vol. 162, Springer-Verlag, New York, 1995.

[2] Artin, Michael, Algebra, Prentice Hall, New York, 1991.

[3] Beachy, J. A., and W. D. Blair, Abstract Algebra, 2nd Ed., Waveland Press, Prospect Heights, Ill., 1996.

[4] Bourbaki, Nicolas, Elements of Mathematics: Algebra I, New York: Springer-Verlag, 1998.
[5] Dixon, G.M., Division Algebras: Octonions Quaternions Complex Numbers and the Algebraic Design of Physics, December 2010, Kluwer Academic Publishers, Dordrecht.

[6] Ellis, G., Rings and Fields, Oxford University Press, 1993.

[7] Hideyuki Matsumura, Commutative Ring Theory, Cambridge University Press, Cambridge, 1986

[8] Hungerford, T., Algebra, Graduate Texts in Mathematics, Vol. 73, Springer-Verlag, New York, 1974.

[9] I. N. Herstein, Topics in Algebra, Wiley Eastern Limited, New Delhi, 2001

[10] Jacobson, N., Basic Algebra I, 2nd Ed., W. H. Freeman \& Company Publishers, San Francisco, 1985.

[11] Jacobson, N., Basic Algebra II, 2nd Ed., W. H. Freeman \& Company Publishers, San Francisco, 1989.

[12] Lam, T. Y., Exercises in Classical Ring Theory, Problem Books in Mathematics, Springer-Verlag, New York, 1995.

[13] Lang, Serge, Undergraduate Algebra (3rd ed.), Berlin, New York: Springer-Verlag, 2005.

[14] Nathan Jacobson, The Theory of Rings. American Mathematical Society Mathematical Surveys, Vol. I. American Mathematical Society, New York, 1943.

[15] Pierce, Richard S., Associative algebras. Graduate Texts in Mathematics, 88. Studies in the History of Modern Science, 9. Springer-Verlag, New York-Berlin, 1982.

[16] Ranjit Biswas, Region Algebra, Theory of Objects \& Theory of Numbers, International Journal of Algebra, Vol. 6 (28) 2012 page 1371-1417.

[17] Ranjit Biswas, Calculus Space, International Journal of Algebra, Vol. 7, 2013, No.16, 791-801.

[18] Van der Waerden, B. L., Algebra, Springer-Verlag, New York, 1991.

[19] Van der Waerden, Bartel Leendert, Algebra, Berlin, New York: Springer-Verlag, 1993, ISBN 978-3-540-56799-8

[20] Walter Rudin, Real and Complex Analysis, McGraw Hills Education, India, August' 2006. 